\title{
Global well-posedness for the Schrödinger equation coupled to a nonlinear oscillator
}

\author{
ALEXANDER KOMECH * \\ Faculty of Mathematics, Vienna University, Wien A-1090, Austria \\ ANDREW KOMECH ${ }^{\dagger}$ \\ Mathematics Department, Texas A\&M University, College Station, TX, USA
}

April 27, 2007

\begin{abstract}
The Schrödinger equation with the nonlinearity concentrated at a single point proves to be an interesting and important model for the analysis of long-time behavior of solutions, such as the asymptotic stability of solitary waves and properties of weak global attractors. In this note, we prove global well-posedness of this system in the energy space $H^{1}$.
\end{abstract}

\section{Introduction and main results}

We are going to prove the well-posedness in $H^{1}$ for the nonlinear Schrödinger equation with the nonlinearity concentrated at a single point:

$$
i \dot{\psi}(x, t)=-\psi^{\prime \prime}(x, t)-\delta(x) F(\psi(0, t)), \quad x \in \mathbb{R},
$$

where the dots and the primes stand for the partial derivatives in $t$ and $x$, respectively. The equation describes the Schrödinger field coupled to a nonlinear oscillator. This equation is a convenient playground for developing the tools for the analysis of long-time behavior of solutions to $U(1)$-invariant Hamiltonian systems with dispersion. The asymptotic stability of the solitary manifold for equation (1.1) has been considered in [BKKS07]. Here we complete this result, giving the proof of the global well-posedness of (1.1) in the energy space.

Let us mention that for the Klein-Gordon equation with the nonlinearity of the same type the global attraction was addressed in [KK06], [KK07].

We assume that

$$
F(\psi)=-\nabla_{\psi} U(\psi), \quad \psi \in \mathbb{C},
$$

for some real-valued potential $U \in C^{2}(\mathbb{C})$, where $\nabla_{\psi}$ is the real derivative with respect to $(\operatorname{Re} \psi, \operatorname{Im} \psi)$. Equation 1.1 is a Hamiltonian system with the Hamiltonian

$$
\mathscr{H}(\psi)=\int_{\mathbb{R}} \frac{\left|\psi^{\prime}(x)\right|^{2}}{2} d x+U(\psi(0)), \quad \psi \in H^{1}=H^{1}(\mathbb{R}) .
$$

\footnotetext{
* On leave from Department of Mechanics and Mathematics, Moscow State University, Moscow 119992, Russia. Supported in part by DFG grant 436 RUS 113/615/0-1, FWF grant P19138-N13, Max-Planck Institute for Mathematics in the Sciences (Leipzig) and Alexander von Humboldt Research Award (2006).

${ }^{\dagger}$ Supported in part by Max-Planck Institute for Mathematics in the Sciences (Leipzig) and by the NSF Grants DMS-0434698 and DMS-0600863.
} 
The Hamiltonian form of (1.1) is

$$
\dot{\Psi}=J D \mathscr{H}(\Psi)
$$

where

$$
\Psi=\left[\begin{array}{c}
\operatorname{Re} \psi \\
\operatorname{Im} \psi
\end{array}\right], \quad J=\left[\begin{array}{cc}
0 & 1 \\
-1 & 0
\end{array}\right],
$$

and $D \mathscr{H}$ is the Fréchet derivative on the Hilbert space $H^{1}$. The value of the Hamiltonian functional is conserved for classical finite energy solutions of (1.1). We assume that equation (1.1) possesses $U(1)$-symmetry, thus requiring that

$$
U(\psi)=u\left(|\psi|^{2}\right), \quad \psi \in \mathbb{C} .
$$

It then follows that $F(0)=0$ and $F\left(e^{i s} \psi\right)=e^{i s} F(\psi)$ for $\psi \in \mathbb{C}, s \in \mathbb{R}$, and that

$$
F(\psi)=a\left(|\psi|^{2}\right) \psi, \quad \psi \in \mathbb{C}, \quad \text { where } \quad a(\cdot)=2 u^{\prime}(\cdot) \in \mathbb{R} .
$$

This symmetry implies that $e^{i \theta} \psi(x, t)$ is a solution to 1.1) if $\psi(x, t)$ is. According to the Nöther theorem, the $U(1)$-invariance leads (formally) to the conservation of the charge, given by the functional

$$
Q(\psi)=\frac{1}{2} \int_{\mathbb{R}}|\psi|^{2} d x
$$

We also assume that $U(\psi)$ is such that

$$
U(z) \geq A-B|z|^{2} \quad \text { with some } \quad A \in \mathbb{R}, \quad B>0 .
$$

We will show that equation (1.1) is globally well-posed in $H^{1}$. We will consider the solutions of class $\psi \in$ $C_{b}(\mathbb{R} \times \mathbb{R})$. All the derivatives in equation (1.1) are understood in the sense of distributions.

Theorem 1.1 (Global well-posedness). Let the conditions (1.2), (1.6) and (1.9) hold with $U \in C^{2}(\mathbb{C})$. Then

(i) For any $\phi \in H^{1}(\mathbb{R})$, the equation for (1.1) with the initial data $\left.\psi\right|_{t=0}=\phi$ has a unique solution $\psi \in$ $C\left(\mathbb{R}, H^{1}(\mathbb{R})\right)$.

(ii) The values of the charge and energy functionals are conserved:

$$
Q(\psi(t))=Q(\phi), \quad \mathscr{H}(\psi(t))=\mathscr{H}(\phi), \quad t \in \mathbb{R} .
$$

(iii) There exists $\Lambda(\phi)>0$ such that the following a priori bound holds:

$$
\sup _{t \in \mathbb{R}}\|\psi(t)\|_{H^{1}} \leq \Lambda(\phi)<\infty
$$

(iv) The map $\mathbf{U}: \psi(0) \mapsto \psi$ is continuous from $H^{1}$ to $L^{\infty}\left([0, T], H^{1}(\mathbb{R})\right)$, for any $T>0$.

Theorem 1.2. Under conditions of Theorem $1.1 \psi \in C^{(1 / 4)}(\mathbb{R} \times \mathbb{R})$.

Let us give the outline of the proof. We need a small preparation first: We show that, without loss of generality, it suffices to prove the theorem assuming that $U$ is uniformly bounded together with its derivatives. Indeed, the a priori bounds on the $L^{\infty}$-norm of $\psi$ imply that the nonlinearity $F(z)$ may be modified for large values of $|z|$. Then we will prove the existence and uniqueness of the solution $\psi \in C_{b}(\mathbb{R} \times[0, \tau])$, for some $\tau>0$. This is accomplished in Section 2 . 
In Section 3, we construct approximate solutions $\psi_{\varepsilon} \in C_{b}\left(\mathbb{R}, H^{1}(\mathbb{R})\right)$ that are solutions to a regularized problem (the $\delta$-function substituted by its smooth approximations $\rho_{\varepsilon}, \varepsilon>0$ ). On one hand, the approximate solutions have their energy and charge conserved. On the other hand, we will show in Section 4 that the approximate solutions converge to $\psi(x, t)$ uniformly for $|x| \leq R, 0 \leq t \leq \tau$.

In Section 5] we use the uniform convergence of approximate solutions to conclude that $\psi \in L^{\infty}\left([0, \tau], H^{1}(\mathbb{R})\right)$ and moreover that $\psi$ could be extended to all $t \geq 0$. Then we show that the energy and the charge are conserved. We will use these conservations to extend the solution $\psi(x, t)$ for $t \in \mathbb{R}$. Then we prove that $\psi \in C\left(\mathbb{R}, H^{1}(\mathbb{R})\right)$.

In Section 6 , we study the Hölder continuity in time, showing that $\psi \in C^{(1 / 4)}(\mathbb{R} \times \mathbb{R})$.

\section{Local well-posedness in $C_{b}$}

Lemma 2.1. A priori bound (1.11) follows from (1.9) and the energy and charge conservation (1.10).

Proof. Let $A \in \mathbb{R}, B>0$ be constants from (1.9), and let $\psi \in H^{1}(\mathbb{R})$. To estimate $\|\psi\|_{H^{1}}$ in terms of the values of $Q(\psi)$ and $\mathscr{H}(\psi)$, we need to control the possibly negative contribution of $U(\psi)$ into $\mathscr{H}(\psi)$. We achieve this control by using the inequality

$$
B|\psi(0)|^{2} \leq B\left[\int_{\mathbb{R}} \hat{\psi}(k) \frac{d k}{2 \pi}\right]^{2} \leq B \int_{\mathbb{R}}\left(B^{2}+\frac{k^{2}}{4}\right)|\hat{\psi}(k)|^{2} \frac{d k}{2 \pi} \cdot \int_{R} \frac{d k}{2 \pi\left(B^{2}+\frac{k^{2}}{4}\right)}=B^{2}\|\psi\|_{L^{2}}^{2}+\frac{1}{4}\left\|\psi^{\prime}\right\|_{L^{2}}^{2} .
$$

This allows us to write

$$
\mathscr{H}(\psi) \geq \frac{1}{2}\left\|\psi^{\prime}\right\|_{L^{2}}^{2}+A-B|\psi(0)|^{2} \geq \frac{1}{4}\left\|\psi^{\prime}\right\|_{L^{2}}^{2}+A-B^{2}\|\psi\|_{L^{2}}^{2}=\frac{1}{4}\|\psi\|_{H^{1}}^{2}+A-\left(B^{2}+\frac{1}{4}\right)\|\psi\|_{L^{2}}^{2} .
$$

The first inequality follows from (1.9), while the second one holds due to the bound (2.1). We rewrite (2.2) as the bound on $\|\psi\|_{H^{1}}^{2}$ :

$$
\|\psi\|_{H^{1}}^{2} \leq\left(8 B^{2}+2\right) Q(\psi)+4 \mathscr{H}(\psi)-4 A .
$$

When we take into account the energy and charge conservation (1.10), the inequality 2.3 leads to the bound (1.11) with

$$
\Lambda(\phi)=\sqrt{\left(8 B^{2}+2\right) Q(\phi)+4 \mathscr{H}(\phi)-4 A} .
$$

Lemma 2.2. Let us assume that Theorem 1.1 is true for the nonlinearities $U$ that satisfy the following additional condition:

$$
\text { For } k=0,1,2 \text { there exist } U_{k}<\infty \quad \text { so that } \sup _{z \in \mathbb{C}}\left|\nabla^{k} U(z)\right| \leq U_{k} .
$$

Then Theorem 1.1 is also true without this additional condition.

Proof. Fix a nonlinearity $U$ that does not necessarily satisfy $\left(2.5\right.$. For a particular initial data $\phi \in H^{1}(\mathbb{R})$ in Theorem 1.1, we choose $\widetilde{U}(z) \in C^{2}(\mathbb{C})$ so that $\widetilde{U}(z)=\widetilde{U}(|z|)$ for $z \in \mathbb{C}$ and $\widetilde{U}(z)=U(z)$ for $|z| \leq \Lambda(\phi)$, where $\Lambda(\phi)$ is defined by (2.4). We can choose $\widetilde{U}$ so that it satisfies (1.9) with the same $A, B$ as $U$ does, and also satisfies the uniform bounds

$$
\sup _{z \in \mathbb{C}}\left|\nabla^{k} \widetilde{U}(z)\right|<\infty, \quad k=0,1,2 .
$$

By the assumption of the Lemma, Theorem 1.1 is true for the nonlinearity $\widetilde{F}=-\nabla \widetilde{U}$ instead of $F=-\nabla U$. Hence, there is a unique solution $\psi(x, t) \in L^{\infty}\left(\mathbb{R}, H^{1}\right) \cap C_{b}(\mathbb{R} \times \mathbb{R})$ to the equation

$$
i \dot{\psi}(x, t)=-\psi^{\prime \prime}(x, t)-\delta(x) \widetilde{F}(\psi(0, t)),
$$


with $\left.\psi\right|_{t=0}=\phi$. By Lemma 2.1, $\psi$ satisfies the a priori bound (1.11) with $\Lambda(\phi)$ defined by (2.4). This bound implies that $|\psi(0, t)| \leq \Lambda(\phi)$ for $t \in \mathbb{R}$. Therefore, $\widetilde{F}(\psi(0, t))=F(\psi(0, t))$ for $t \in \mathbb{R}$, and $\psi(x, t)$ is also a solution to (1.1) with the nonlinearity $F=-\nabla U$.

From now on, we shall assume in the proof of Theorem 1.1 that the bounds (2.5) hold true.

Lemma 2.3. (i) Let $\phi \in H^{1}:=H^{1}(\mathbb{R})$. There exists $\tau>0$ that depends only on $U_{2}$ in (2.5) so that there is a unique solution $\psi \in C_{b}(\mathbb{R} \times[0, \tau])$ to equation (1.1) with the initial data $\left.\psi\right|_{t=0}=\phi$.

(ii) The map $\phi \mapsto \psi$ is continuous from $H^{1}$ to $C_{b}(\mathbb{R} \times[0, \tau])$.

Proof. Let us denote the dynamical group for the free Schrödinger equation by

$$
\mathbf{W}_{t} \phi(x)=\frac{1}{\sqrt{2 \pi t}} \int_{\mathbb{R}} e^{i \frac{|x-y|^{2}}{2 t}} \phi(y) d y, \quad x \in \mathbb{R} .
$$

For its Fourier transform, we have:

$$
\mathscr{F}_{x \rightarrow k}\left[\mathbf{W}_{t} \phi(x)\right](k)=e^{i k^{2} t} \hat{\phi}(k), \quad k \in \mathbb{R} .
$$

Then the solution $\psi$ to (1.1) with the initial data $\left.\psi\right|_{t=0}=\phi$ admits the Duhamel representation

$$
\psi(x, t)=\mathbf{W}_{t} \phi(x)=\mathbf{W}_{t} \phi(x)+\mathbf{Z} \psi(x, t),
$$

where

$$
\mathbf{Z} \psi(x, t)=-\int_{0}^{t} \mathbf{W}_{s} \boldsymbol{\delta}(x) F(\psi(0, t-s)) d s=-\int_{0}^{t} \frac{e^{i \frac{x^{2}}{2 s}}}{\sqrt{2 \pi s}} F(\psi(0, t-s)) d s .
$$

The Fourier representation (2.7) implies that $\mathbf{W}_{t} \phi(x) \in C_{b}\left(\mathbb{R}, H^{1}\right) \subset C_{b}(\mathbb{R} \times \mathbb{R})$. Further, we compute for $\psi_{1}$, $\psi_{2} \in C_{b}(\mathbb{R} \times[0, \tau]):$

$$
\left|\mathbf{Z} \psi_{2}(x, t)-\mathbf{Z} \psi_{1}(x, t)\right| \leq \int_{0}^{t} \frac{\left|F\left(\psi_{2}(0, t-s)\right)-F\left(\psi_{1}(0, t-s)\right)\right|}{\sqrt{2 \pi s}} d s \leq U_{2} \sqrt{t} \sup _{0 \leq s \leq t}\left|\psi_{2}(s)-\psi_{1}(s)\right|,
$$

where we used (2.5) with $k=2$. For definiteness, we set

$$
\tau=\frac{1}{4 U_{2}^{2}} .
$$

Then the map $\psi \mapsto \mathbf{W}_{t} \phi+\mathbf{Z} \psi$ is contracting in the space $C_{b}(\mathbb{R} \times[0, \tau])$. It follows that equation (2.8) admits a unique solution $\psi \in C_{b}(\mathbb{R} \times[0, \tau])$, proving the first part of the theorem. The second part of the theorem also follows by contraction.

\section{Regularized equation}

We proved that there is a unique solution $\psi(x, t) \in C([0, \tau] \times \mathbb{R})$. Now we are going to prove that $\psi \in$ $L^{\infty}\left(\mathbb{R}_{+}, H^{1}\right)$ and moreover that $\|\psi(t)\|_{H^{1}}$ is bounded uniformly in time.

Let us fix a family of functions $\rho_{\varepsilon}(x)$ approximating the Dirac $\delta$-function. We pick $\rho_{1}(x) \in C_{0}^{\infty}[-1,1]$, nonnegative, and such that $\int_{\mathbb{R}} \rho_{1}(x) d x=1$, and define

$$
\rho_{\varepsilon}(x)=\frac{1}{\varepsilon} \rho_{1}\left(\frac{x}{\varepsilon}\right), \quad \varepsilon \in(0,1)
$$


so that

$$
\operatorname{supp} \rho_{\varepsilon}(x) \subseteq[-\varepsilon, \varepsilon], \quad \rho_{\varepsilon}(x) \geq 0, \quad \int_{\mathbb{R}} \rho_{\varepsilon}(x) d x=1 .
$$

Consider the smoothed equation with the "mean field interaction"

$$
i \dot{\psi}(x, t)=-\Delta \psi(x, t)-\rho_{\varepsilon}(x) F\left(\left\langle\rho_{\varepsilon}, \psi(t)\right\rangle\right),
$$

where

$$
\left\langle\rho_{\varepsilon}, \psi(t)\right\rangle=\left\langle\rho_{\varepsilon}(\cdot), \psi(\cdot, t)\right\rangle=\int_{\mathbb{R}} \rho_{\varepsilon}(x) \psi(x, t) d x .
$$

Clearly, equation (3.2) is the Hamiltonian equation, with the Hamilton functional

$$
\mathscr{H}_{\varepsilon}(\psi)=\int \frac{|\nabla \psi|^{2}}{2} d x+U\left(\left\langle\rho_{\varepsilon}, \psi\right\rangle\right)
$$

The Hamiltonian form of (3.2) is (cf. (1.4)

$$
\dot{\Psi}_{\varepsilon}=J D \mathscr{H}_{\varepsilon}\left(\Psi_{\varepsilon}\right) .
$$

The solution $\psi_{\varepsilon}$ to (3.2) with the initial data $\left.\psi_{\varepsilon}\right|_{t=0}=\phi$ admits the Duhamel representation

$$
\psi_{\varepsilon}(x, t)=\mathbf{W}_{t} \phi(x)+\mathbf{Z}_{\varepsilon} \psi_{\varepsilon}(x, t),
$$

where

$$
\mathrm{Z}_{\varepsilon} \psi_{\varepsilon}(x, t)=-\int_{0}^{t} \mathbf{W}_{s} \rho_{\varepsilon}(x) F\left(\left\langle\rho_{\varepsilon}, \psi_{\varepsilon}(t-s)\right\rangle\right) d s .
$$

Lemma 3.1 (Local well-posedness). (i) For any $\varepsilon \in(0,1)$, there exists $\tau_{\varepsilon}>0$ that depends on $\varepsilon$ and on $U_{2}$ from (2.5) so that there is a unique solution $\psi_{\varepsilon} \in C_{b}\left(\left[0, \tau_{\varepsilon}\right], H^{1}\right)$ to equation (3.2) with $\left.\psi_{\varepsilon}\right|_{t=0}=\phi$.

(ii) For each $t \leq \tau_{\varepsilon}$, the map $\mathrm{U}_{\varepsilon}(t): \phi=\psi_{\varepsilon}(0) \mapsto \psi_{\varepsilon}(t)$ is continuous in $H^{1}$.

(iii) The values of the functionals $\mathscr{H}_{\varepsilon}$ and $Q$ on solutions to (3.2) are conserved in time.

Proof. (i) For $\psi_{1}, \psi_{2} \in C_{b}\left(\left[0, \tau_{\varepsilon}\right], H^{1}\right)$, we compute:

$$
\begin{aligned}
& \left\|\mathbf{Z}_{\varepsilon} \psi_{2}(\cdot, t)-\mathbf{Z}_{\varepsilon} \psi_{1}(\cdot, t)\right\|_{H^{1}} \\
& =\left\|\int_{0}^{t} \mathbf{W}_{s} \rho_{\varepsilon} F\left(\left\langle\rho_{\varepsilon}, \psi_{2}(t-s)\right\rangle\right)-F\left(\left\langle\rho_{\varepsilon}, \psi_{1}(t-s)\right\rangle\right) d s\right\|_{H^{1}} \\
& \leq \int_{0}^{t}\left\|\mathbf{W}_{s} \rho_{\varepsilon}\right\|_{H^{1}}\left|F\left(\left\langle\rho_{\varepsilon}, \psi_{2}(t-s)\right\rangle\right)-F\left(\left\langle\rho_{\varepsilon}, \psi_{1}(t-s)\right\rangle\right)\right| d s .
\end{aligned}
$$

The first factor under the integral sign is bounded uniformly for $0<s \leq t$ :

$$
\left\|\mathbf{W}_{s} \rho_{\varepsilon}\right\|_{H_{x}^{1}}=\frac{1}{\sqrt{2 \pi}}\left\|\sqrt{1+k^{2}} e^{i k^{2} s / 2} \widehat{\rho_{\varepsilon}}(k)\right\|_{L_{k}^{2}}=\left\|\rho_{\varepsilon}\right\|_{H^{1}} .
$$

Taking this into account, we get:

$$
\begin{aligned}
\left\|\mathbf{Z}_{\varepsilon} \psi_{2}(\cdot, t)-\mathbf{Z}_{\varepsilon} \psi_{1}(\cdot, t)\right\|_{H^{1}} & \leq\left\|\rho_{\varepsilon}\right\|_{H^{1}} \int_{0}^{t}\left|F\left(\left\langle\rho_{\varepsilon}, \psi_{2}(t-s)\right\rangle\right)-F\left(\left\langle\rho_{\varepsilon}, \psi_{1}(t-s)\right\rangle\right)\right| d s \\
& \leq t U_{2}\left\|\rho_{\varepsilon}\right\|_{H^{1}} \sup _{s \in[0, t]}\left|\left\langle\rho_{\varepsilon}, \psi_{2}(s)-\psi_{1}(s)\right\rangle\right| .
\end{aligned}
$$

Therefore, the map $\psi \mapsto \mathbf{W}_{t} \phi+\mathbf{Z}_{\varepsilon} \psi$ is contracting if we choose, for definiteness,

$$
\tau_{\varepsilon}=\frac{1}{4 U_{2}\left\|\rho_{\varepsilon}\right\|_{H^{1}}} .
$$


(ii) The continuity of the mapping $\mathbf{U}_{\varepsilon}(t)$ also follows from the contraction argument.

(iii) It suffices to prove the conservation of the values of $\mathscr{H}_{\varepsilon}\left(\psi_{\varepsilon}(t)\right)$ and $Q\left(\psi_{\varepsilon}(t)\right)$ for $\phi \in H^{2}:=H^{2}(\mathbb{R})$ since the functionals are continuous on $H^{1}$. For $\phi \in H^{2}$, the corresponding solution belongs to the space $C_{b}\left(\left[0, \tau_{\varepsilon}\right], H^{2}\right)$ by the Duhamel representation (3.5). Then the energy and charge conservation follows by the Hamiltonian structure (3.4). Namely, the differentiation of the Hamilton functional gives by the chain rule,

$$
\frac{d}{d t} \mathscr{H}_{\varepsilon}\left(\Psi_{\varepsilon}(t)\right)=\left\langle D \mathscr{H}_{\varepsilon}\left(\Psi_{\varepsilon}(t)\right), \dot{\Psi}_{\varepsilon}(t)\right\rangle=\left\langle D \mathscr{H}_{\varepsilon}\left(\Psi_{\varepsilon}(t)\right), J D \mathscr{H}_{\varepsilon}\left(\Psi_{\varepsilon}(t)\right)\right\rangle=0
$$

since the Fréchet derivative $\left.D \mathscr{H}_{\varepsilon}\left(\Psi_{\varepsilon}(t)\right)=-\Delta \Psi_{\varepsilon}(\cdot, t)-\rho_{\varepsilon}(\cdot) F\left(\left\langle\rho_{\varepsilon}, \Psi_{\varepsilon}(t)\right)\right\rangle\right)$ belongs to $L^{2}(\mathbb{R})$ for $t \in$ $\left[0, \tau_{\varepsilon}\right]$. Similarly, the charge conservation follows by the differentiation,

$$
\begin{aligned}
\frac{d}{d t} Q\left(\Psi_{\varepsilon}(t)\right) & =\left\langle D Q\left(\Psi_{\varepsilon}(t)\right), \dot{\Psi}_{\varepsilon}(t)\right\rangle=\left\langle D Q\left(\Psi_{\varepsilon}(t)\right), J D \mathscr{H}_{\varepsilon}\left(\Psi_{\varepsilon}(t)\right)\right\rangle \\
& =\left\langle\Psi_{\varepsilon}(x, t), J \Delta \Psi_{\varepsilon}(x, t)\right\rangle-\left\langle\Psi_{\varepsilon}(x, t), J \rho_{\varepsilon}(x) F\left(\left\langle\rho_{\varepsilon}, \Psi_{\varepsilon}(t)\right\rangle\right)\right\rangle .
\end{aligned}
$$

Here $\left.\left.\Psi_{\varepsilon}(x, t), J \Delta \Psi_{\varepsilon}(x, t)\right\rangle=\nabla \Psi_{\varepsilon}(x, t), J \nabla \Psi_{\varepsilon}(x, t)\right\rangle=0$, and also

$$
\begin{aligned}
\left\langle\Psi_{\varepsilon}(x, t), J \rho_{\varepsilon}(x) F\left(\left\langle\rho_{\varepsilon}, \Psi_{\varepsilon}(t)\right\rangle\right)\right\rangle & =\int \Psi_{\varepsilon}(x, t) \cdot\left[J \rho_{\varepsilon}(x) F\left(\left\langle\rho_{\varepsilon}, \Psi_{\varepsilon}(t)\right\rangle\right)\right] d x \\
& =\left\langle\rho_{\varepsilon}, \Psi_{\varepsilon}(t)\right\rangle \cdot\left[J F\left(\left\langle\rho_{\varepsilon}, \Psi_{\varepsilon}(t)\right\rangle\right)\right]=0 .
\end{aligned}
$$

Here "." stands for the real scalar product in $\mathbb{R}^{2}$, and $Z \cdot[J F(Z)]=0$ for $Z \in \mathbb{R}^{2}$ since $F(Z)=a(|Z|) Z$ with $a(|Z|) \in \mathbb{R}$ by (1.7).

Corollary 3.2 (Global well-posedness). to equation (3.2) with $\left.\psi_{\varepsilon}\right|_{t=0}=\phi$.

(i) For any $\varepsilon>0$, $\varepsilon \leq 1$, there exists a unique solution $\psi_{\varepsilon} \in C\left(\mathbb{R}, H^{1}\right)$

The $H^{1}$-norm of $\psi_{\varepsilon}$ is bounded uniformly in time:

$$
\sup _{t \in \mathbb{R}}\left\|\psi_{\varepsilon}(t)\right\|_{H^{1}} \leq \Lambda_{\varepsilon}(\phi), \quad t \in \mathbb{R},
$$

where

$$
\Lambda_{\varepsilon}(\phi)=\sqrt{\left(8 B^{2}+2\right) Q(\phi)+4 \mathscr{H}_{\varepsilon}(\phi)-4 A} .
$$

(ii) For each $t \geq 0$, the map $\mathrm{U}_{\mathcal{\varepsilon}}(t): \psi_{\varepsilon}(0) \mapsto \psi_{\varepsilon}(t)$ is continuous in $H^{1}$.

Proof. (i) The existence and uniqueness of the solution $\psi_{\varepsilon} \in C_{b}\left(\left[0, \tau_{\varepsilon}\right], H^{1}\right)$ follow from Lemma $3.1(i)$. The bound on the value of the $H^{1}$-norm of $\psi_{\varepsilon}(t)$ is obtained as in Lemma2.1 Namely, noting that

$$
U\left(\left\langle\rho, \psi_{\varepsilon}\right\rangle\right) \geq A-B\left\langle\rho, \psi_{\varepsilon}\right\rangle^{2} \geq A-B \sup _{x \in \mathbb{R}}\left|\psi_{\varepsilon}\right|^{2} \geq A-B^{2}\|\psi\|_{L^{2}}^{2}-\frac{1}{4}\left\|\psi^{\prime}\right\|_{L^{2}}^{2}
$$

and using the energy and charge conservation proved in Lemma3.1(iii), we conclude that

$$
\left(2 B^{2}+\frac{1}{2}\right) Q(\phi)+\mathscr{H}_{\varepsilon}(\phi)=\left(2 B^{2}+\frac{1}{2}\right) Q\left(\psi_{\varepsilon}\right)+\mathscr{H}_{\varepsilon}\left(\psi_{\varepsilon}\right) \geq A+\frac{1}{4}\left\|\psi_{\varepsilon}\right\|_{H^{1}}^{2},
$$

so that

$$
\left\|\psi_{\varepsilon}\right\|_{H^{1}}^{2} \leq\left(8 B^{2}+2\right) Q(\phi)+4 \mathscr{H}_{\varepsilon}(\phi)-4 A \text {. }
$$


By (3.7), the time span $\tau_{\varepsilon}$ depends only on $\left\|\rho_{\varepsilon}\right\|_{H^{1}}$ and $U_{2}$. Hence, the bound (3.11) allows us to extend the solution to $t \in\left[\tau_{\varepsilon}, 2 \tau_{\varepsilon}\right]$. The bound (3.11) for $t \in\left[0,2 \tau_{\varepsilon}\right]$ follows from (3.13) by the energy and charge conservation proved in Lemma 3.1 (iii). We conclude by induction that the solution exists and the bound (3.11) holds for all $t \in \mathbb{R}$.

(ii) The continuity of the mapping $\mathrm{U}_{\mathcal{\varepsilon}}(t): \psi_{\varepsilon}(0) \mapsto \psi_{\varepsilon}(t)$ for all $t \geq 0$ follows from its continuity for small times by dividing the interval $[0, t]$ into small time intervals.

\section{Convergence of regularized solutions}

Lemma 4.1. Let $\tau$ and $\psi \in C_{b}(\mathbb{R} \times[0, \tau])$ be as in Lemma 2.3 and let $\psi_{\varepsilon} \in C\left(\mathbb{R}_{+}, H^{1}\right)$ be as in Corollary 3.2 Then for any finite $R>0$

$$
\psi_{\varepsilon}(x, t) \underset{\varepsilon \rightarrow 0}{\rightrightarrows} \psi(x, t), \quad|x| \leq R, \quad 0 \leq t \leq \tau
$$

Proof. We have

$$
\begin{array}{r}
\psi_{\varepsilon}(x, t)=W_{t} \phi(x)+\int_{0}^{t} \mathbf{W}_{s} \rho_{\varepsilon}(x) F\left(\left\langle\rho_{\varepsilon}, \psi_{\varepsilon}(t-s)\right\rangle\right) d s, \\
\psi(x, t)=W_{t} \phi(x)+\int_{0}^{t} \mathbf{W}_{s} \delta(x) F(\psi(0, t-s)) d s .
\end{array}
$$

Taking the difference of these equations and regrouping the terms, we can write:

$$
\begin{array}{r}
\psi_{\varepsilon}(x, t)-\psi(x, t)=\int_{0}^{t} \mathbf{W}_{s} \rho_{\varepsilon}(x)\left(F\left(\left\langle\rho_{\varepsilon}, \psi_{\varepsilon}(t-s)\right\rangle\right)-F(\psi(0, t-s))\right) d s \\
+\int_{0}^{t}\left[\mathbf{W}_{s} \rho_{\varepsilon}(x)-\mathbf{W}_{s} \delta(x)\right] F(\psi(0, t-s)) d s .
\end{array}
$$

Let us analyze the first term in the right-hand side of (4.4). It is bounded by

$$
\begin{array}{r}
\left|\int_{0}^{t} \frac{e^{i \frac{(x-y)^{2}}{2 s}}}{\sqrt{2 \pi s}} \rho_{\varepsilon}(y) d y d s\right| \sup _{0 \leq s \leq t}\left|F\left(\left\langle\rho_{\varepsilon}, \psi_{\varepsilon}(s)\right\rangle\right)-F(\psi(0, s))\right| \\
\leq\left|\int_{0}^{t} \frac{d s}{\sqrt{2 \pi s}}\right| U_{2} \sup _{|x| \leq \varepsilon, 0 \leq s \leq t}\left|\psi_{\varepsilon}(x, s)-\psi(x, s)\right| \\
\leq \sqrt{\frac{2 t}{\pi}} U_{2} \sup _{|x| \leq \varepsilon, 0 \leq s \leq t}\left|\psi_{\varepsilon}(x, s)-\psi(x, s)\right| \\
\leq \frac{1}{2} \sup _{|x| \leq \varepsilon, 0 \leq s \leq t}\left|\psi_{\varepsilon}(x, s)-\psi(x, s)\right|,
\end{array}
$$

where in the last inequality we used (3.7). Setting $M_{R, \tau}=\sup _{|x| \leq R, 0 \leq t \leq \tau}\left|\psi_{\varepsilon}(x, t)-\psi(x, t)\right|$, we can rewrite (4.4) as

$$
M_{R, \tau} \leq \frac{1}{2} M_{R, \tau}+\sup _{|x| \leq R, 0 \leq t \leq \tau} \int_{0}^{t}\left[\mathbf{W}_{s} \rho_{\varepsilon}(x)-\mathbf{W}_{s} \boldsymbol{\delta}(x)\right] F(\psi(0, t-s)) d s .
$$

Therefore,

$$
M_{R, \tau} \leq 2 \sup _{|x| \leq R, 0 \leq t \leq \tau} \int_{0}^{t} \int \frac{e^{i \frac{(x-y)^{2}}{2 s}}}{\sqrt{2 \pi s}}\left[\rho_{\varepsilon}(y)-\delta(y)\right] d y F(\psi(0, t-s)) d s .
$$


Alexander Komech, Andrew Komech: Schrödinger equation coupled to nonlinear oscillator

We claim that the right-hand side tends to zero as $\varepsilon \rightarrow 0$. To prove this, we split the integral into two pieces:

$$
\begin{aligned}
& I_{1}(\delta, \varepsilon)=\int_{\delta}^{t} \int \frac{e^{i \frac{(x-y)^{2}}{2 s}}}{\sqrt{2 \pi s}}\left[\rho_{\varepsilon}(y)-\delta(y)\right] d y F(\psi(0, t-s)) d s, \\
& I_{2}(\delta, \varepsilon)=\int_{0}^{\delta} \int \frac{e^{i \frac{(x-y)^{2}}{2 s}}}{\sqrt{2 \pi s}}\left[\rho_{\varepsilon}(y)-\delta(y)\right] d y F(\psi(0, t-s)) d s,
\end{aligned}
$$

where $\delta \in(0, t)$ is yet to be chosen. Let us analyze the term (4.7):

$$
\left|I_{1}(\delta, \varepsilon)\right| \leq C U_{0} \sup _{s \geq \delta,|x| \leq R}\left|\int_{|y|<\varepsilon} \frac{e^{i \frac{(x-y)^{2}}{2 s}}}{\sqrt{2 \pi s}}\left[\rho_{\varepsilon}(y)-\delta(y)\right] d y\right| .
$$

Since $s \geq \delta>0$ and $|x| \leq R$, the function $\frac{e^{i \frac{(x-y)^{2}}{2 s}}}{\sqrt{2 \pi s}}$ is Lipschitz in $y \in[-\varepsilon, \varepsilon]$, uniformly in all the parameters. Therefore,

$$
\int_{\mathbb{R}} \frac{e^{i \frac{(x-y)^{2}}{2 s}}}{\sqrt{2 \pi s}}\left[\rho_{\varepsilon}(y)-\delta(y)\right] d y \rightarrow 0, \quad \varepsilon \rightarrow 0,
$$

uniformly in the parameters. We conclude that

$$
\lim _{\varepsilon \rightarrow 0} I_{1}(\delta, \varepsilon)=0
$$

for any fixed $\delta>0$. We then bound (4.8) uniformly by

$$
I_{2}(\delta, \varepsilon) \leq C U_{0} \int\left(\rho_{\varepsilon}(y)+\delta(y)\right) d y \int_{0}^{\delta} \frac{d s}{\sqrt{s}} \leq C \sqrt{\delta},
$$

with $C$ independent of $\varepsilon$. Now apparently the right-hand side of (4.6) tends to zero as $\varepsilon \rightarrow 0$.

\section{Well-posedness in energy space}

Lemma 5.1 (Local well-posedness). There is a unique solution $\psi \in L^{\infty}\left([0, \tau], H^{1}(\mathbb{R})\right) \cap C_{b}(\mathbb{R} \times[0, \tau])$ to equation (1.1) with $\left.\psi\right|_{t=0}=\phi$, where $\tau$ is as in (2.10).

Proof. The unique solution $\psi \in C_{b}(\mathbb{R} \times[0, \tau])$ is constructed in Lemma 2.3. According to (3.11) and (4.1),

$$
\|\psi(t)\|_{H^{1}} \leq \liminf _{\varepsilon \rightarrow 0}\left\|\psi_{\varepsilon}(t)\right\|_{H^{1}} \leq \Lambda(\phi), \quad 0 \leq t \leq \tau .
$$

Lemma 5.2. The values of the functionals $\mathscr{H}$ and $Q$ are conserved in time for $t \in[0, \tau]$.

Proof. The convergence (4.1) and the bounds 3.11 imply that

$$
Q(\psi(t))=\frac{1}{2}\|\psi(t)\|_{L^{2}}^{2} \leq \frac{1}{2} \lim _{\varepsilon \rightarrow 0}\left\|\psi_{\varepsilon}(t)\right\|_{L^{2}}^{2}=Q(\phi),
$$

where we used the conservation of $Q$ for the approximate solutions $\psi_{\varepsilon}$ (Lemma 3.1). The same argument applied to the initial data $\left.\psi\right|_{t=t_{0}}$ with any $t_{0} \in(0, \tau)$ and combined with the uniqueness of the solution, allows 
to conclude that $Q(\psi(t))$ is monotonically non-increasing when time changes from 0 to $\tau$. Instead, solving the Schrödinger equation backwards in time and using the uniqueness of solution, we can as well conclude that $Q(\psi(t))$ is monotonically non-decreasing when time changes from 0 to $\tau$. This proves that $Q(\psi(t))=$ const for $t \in[0, \tau]$.

To prove the conservation of $\mathscr{H}(\psi(t))$, we will need the relation

$$
\lim _{\varepsilon \rightarrow 0} U\left(\left\langle\rho_{\varepsilon}, \psi_{\varepsilon}\right\rangle\right)=U(\psi(0, t)) .
$$

This relation follows from continuity of the potential $U$ and from

$$
\lim _{\varepsilon \rightarrow 0}\left\langle\rho_{\varepsilon}, \psi_{\varepsilon}(t)\right\rangle=\lim _{\varepsilon \rightarrow 0}\left\langle\rho_{\varepsilon},\left(\psi_{\varepsilon}(t)-\psi(t)\right)\right\rangle+\lim _{\varepsilon \rightarrow 0}\left\langle\rho_{\varepsilon}, \psi(t)\right\rangle=\psi(0, t),
$$

where $\lim _{\varepsilon \rightarrow 0}\left\langle\rho_{\varepsilon},\left(\psi_{\varepsilon}(t)-\psi(t)\right)\right\rangle=0$ since $\psi_{\varepsilon}$ approaches $\psi$ uniformly for $0 \leq t \leq \tau$ and $|x| \leq R$ (including $x=0$ ), while $\lim _{\varepsilon \rightarrow 0}\left\langle\rho_{\varepsilon}, \psi(t)\right\rangle=\psi(0, t)$ since $\psi$ is continuous in $x$ (due to the finiteness of $H^{1}$-norm of $\psi$ that follows from (5.1)). We have:

$$
\mathscr{H}(\psi(t))=\frac{\|\nabla \psi(x, t)\|_{L^{2}}^{2}}{2}+U(\psi(0, t)) \leq \lim _{\varepsilon \rightarrow 0}\left\{\frac{\left\|\nabla \psi_{\varepsilon}(x, t)\right\|_{L^{2}}^{2}}{2}+U\left(\left\langle\rho_{\varepsilon}, \psi_{\varepsilon}\right\rangle\right)\right\}=\mathscr{H}(\phi),
$$

where we used the relation (5.3) and (4.1). We also used the conservation of the values of the functional $\mathscr{H}_{\varepsilon}$ for the approximate solutions $\psi_{\varepsilon}$ (see Lemma 3.1). Proceeding just as with $Q(\psi(t))$ above, we conclude that $\mathscr{H}(\psi(t))=$ const for $0 \leq t \leq \tau$.

Corollary 5.3 (Global well-posedness). There is a unique solution $\psi \in L^{\infty}\left(\mathbb{R}, H^{1}(\mathbb{R})\right) \cap C_{b}(\mathbb{R} \times \mathbb{R})$ to equation (1.1) with $\left.\psi\right|_{t=0}=\phi$. The values of the functionals $\mathscr{H}$ and $Q$ are conserved in time.

Proof. The solution $\psi \in L^{\infty}\left([0, \tau], H^{1}\right)$ constructed in Lemma 5.1 exists for $0 \leq t \leq \tau$, where the time span $\tau$ defined in (2.10) depends only on $U_{2}$ from (2.5). Hence, the bound (1.11) at $t=\tau$ allows us to extend the solution $\psi$ constructed in Lemma 5.1 to the time interval $[\tau, 2 \tau]$. We proceed by induction.

For the conclusion of Theorem 1.1, it remains to prove that $\psi \in C\left(\mathbb{R}, H^{1}(\mathbb{R})\right)$. This follows from the next two lemmas.

Lemma 5.4. $\psi \in C\left(\mathbb{R}, H_{\text {weak }}^{1}(\mathbb{R})\right)$.

Proof. Fix $f \in H^{-1}(\mathbb{R})$ and pick any $\delta>0$. Since $H^{1}$ is dense in $H^{-1}$, there exists $g \in H^{1}(\mathbb{R})$ such that

$$
\|f-g\|_{H^{-1}}<\frac{\delta}{4 \Lambda(\phi)}
$$

where $\Lambda(\phi)$ given by (2.4) is the a priori bound on $\|\psi(t)\|_{H^{1}}$ proved in Lemma2.1 on the grounds of the energy and the charge conservation for $\psi(t)$. Then

$$
\begin{aligned}
& \left|\left\langle f, \psi(t)-\psi\left(t_{0}\right)\right\rangle\right| \leq\left|\left\langle f-g, \psi(t)-\psi\left(t_{0}\right)\right\rangle\right|+\left|\left\langle g, \psi(t)-\psi\left(t_{0}\right)\right\rangle\right| \\
& \leq\|f-g\|_{H^{-1}}\left(\|\psi(t)\|_{H^{1}}+\left\|\psi\left(t_{0}\right)\right\|_{H^{1}}\right)+\|g\|_{H^{1}}\left\|\psi(t)-\psi\left(t_{0}\right)\right\|_{H^{-1}} .
\end{aligned}
$$

By (5.5), the first term in the right-hand side of (5.7) is bounded by $\delta / 2$. By Corollary 5.3 , we have $\psi \in$ $L^{\infty}\left(\mathbb{R}, H^{1}(\mathbb{R})\right)$, and equation (1.1) yields $\psi \in C\left(\mathbb{R}, H^{-1}(\mathbb{R})\right)$. Hence, the second term in the right-hand side of (5.7) becomes smaller than $\delta / 2$ if $t$ is sufficiently close to $t_{0}$. Since $\delta>0$ was arbitrary, this proves that $\lim _{t \rightarrow t_{0}}\left\langle f, \psi(t)-\psi\left(t_{0}\right)\right\rangle=0$. 
Proposition 5.5. $\psi \in C\left(\mathbb{R}, H^{1}(\mathbb{R})\right)$.

Proof. Let us fix $t_{0} \in \mathbb{R}$ and compute

$$
\lim _{t \rightarrow t_{0}}\left\|\psi(t)-\psi\left(t_{0}\right)\right\|_{H^{1}}^{2}=\lim _{t \rightarrow t_{0}}\left(\|\psi(t)\|_{H^{1}}^{2}-2\left\langle\psi(t), \psi\left(t_{0}\right)\right\rangle_{H^{1}}+\left\|\psi\left(t_{0}\right)\right\|_{H^{1}}^{2}\right) .
$$

The relation

$$
\|\psi(t)\|_{H^{1}}^{2}=2(Q(\psi(t))+H(\psi(t)))-2 U(\psi(0, t)),
$$

together with the conservation of the energy and charge and the continuity of $\psi(0, t)$ for $t \in \mathbb{R}$ (see Corollary [5.3), shows that

$$
\lim _{t \rightarrow t_{0}}\|\psi(t)\|_{H^{1}}^{2}=\left\|\psi\left(t_{0}\right)\right\|_{H^{1}}^{2} .
$$

By Lemma 5.4 $\lim _{t \rightarrow t_{0}}\left\langle\psi(t), \psi\left(t_{0}\right)\right\rangle_{H^{1}}=\left\langle\psi\left(t_{0}\right), \psi\left(t_{0}\right)\right\rangle_{H^{1}}$. This shows that the right-hand side of (5.8) is equal to zero.

Now Theorem 1.1 is proved.

\section{Hölder regularity of solution}

In this section, we prove Theorem 1.2

Lemma 6.1. If $\phi \in H^{1}$, then $\mathbf{W}_{(\cdot)} \phi(x) \in C^{(1 / 4)}[0, \tau]$, uniformly in $x \in \mathbb{R}$.

Proof. Let $t, t^{\prime} \in[0, \tau]$. We have by the Cauchy-Schwarz inequality:

$$
\begin{array}{r}
\left|\mathbf{W}_{t^{\prime}} \phi(x)-\mathbf{W}_{t} \phi(x)\right| \leq C\left|\int e^{-i k x}\left(e^{i \frac{t^{\prime} k^{2}}{2}}-e^{i \frac{t k^{2}}{2}}\right) \hat{\phi}(k) d k\right| \\
\leq C \int \min \left(1,\left|t^{\prime}-t\right| k^{2}\right)|\hat{\phi}(k)| d k \leq C\left[\int_{\mathbb{R}} \frac{\min \left(1,\left|t^{\prime}-t\right| k^{2}\right)^{2}}{1+k^{2}} d k\right]^{\frac{1}{2}}\|\phi\|_{H^{1}}
\end{array}
$$

We bound the last integral as follows:

$$
\int_{\mathbb{R}} \frac{\min \left(1,\left|t^{\prime}-t\right| k^{2}\right)^{2}}{1+k^{2}} d k \leq \int_{|k|<\left|t^{\prime}-t\right|^{-\frac{1}{2}}} \frac{\left|t^{\prime}-t\right|^{2} k^{4}}{1+k^{2}} d k+\int_{|k|>\left|t^{\prime}-t\right|^{-\frac{1}{2}}} \frac{d k}{1+k^{2}} \leq \mathrm{const}\left|t^{\prime}-t\right|^{\frac{1}{2}} .
$$

Lemma 6.2 (Regularity of $\psi(0, t))$. The unique solution $\psi \in C_{b}(\mathbb{R} \times[0, \tau])$ to equation (1.1) with the initial data $\left.\psi\right|_{t=0}=\phi$ constructed in Lemma 2.3 satisfies

$$
\psi(0, \cdot) \in C^{(1 / 4)}[0, \tau] .
$$

Proof. Due to Lemma 6.1 it suffices to consider the regularity of $\mathbf{Z} \psi(0, t)$. For any $t, t^{\prime} \in[0, \tau], t^{\prime}<t$, we have:

$$
\mathbf{Z} \psi\left(0, t^{\prime}\right)-\mathbf{Z} \psi(0, t)=\int_{0}^{t}\left[\frac{F(\psi(0, s))}{\sqrt{2 \pi\left(t^{\prime}-s\right)}}-\frac{F(\psi(0, s))}{\sqrt{2 \pi(t-s)}}\right] d s+\int_{t}^{t^{\prime}} \frac{F(\psi(0, s))}{\sqrt{2 \pi\left(t^{\prime}-s\right)}} d s .
$$

The first integral in the right-hand side of (6.1) is bounded by

$$
C_{1} \int_{0}^{t}\left|\frac{1}{\sqrt{t^{\prime}-s}}-\frac{1}{\sqrt{t-s}}\right| d s \leq C_{2}\left|t^{\prime}-t\right|^{1 / 2} .
$$

The second integral in the right-hand side of $(6.1)$ is also bounded by $C\left|t^{\prime}-t\right|^{1 / 2}$. 
Lemma 6.3. $\psi(x, \cdot) \in C^{(1 / 4)}(\mathbb{R})$, uniformly in $x \in \mathbb{R}$.

Proof. We have the relation

$$
\psi(x, t)=\mathbf{W}_{t-t_{0}} \psi\left(x, t_{0}\right)+\int_{0}^{t-t_{0}} \frac{e^{i \frac{i^{2}}{2 s}}}{\sqrt{2 \pi s}} F(\psi(0, t-s)) d s .
$$

By Lemma 6.1 the first term in the right-hand side of 6.2), considered as a function of time, belongs to $C^{(1 / 4)}(\mathbb{R})$ (uniformly in $x \in \mathbb{R}$ ). The second term in the right-hand side of $(6.2)$ is bounded by const $\left|t-t_{0}\right|^{1 / 2}$. This proves that $\psi(x, \cdot) \in C^{(1 / 4)}(\mathbb{R})$, uniformly in $x$.

It remains to mention that the Hölder continuity in $x$ follows from the inclusion $H^{1}(\mathbb{R}) \subset C^{(1 / 4)}(\mathbb{R})$. Theorem 1.2 is proved.

\section{Acknowledgments}

The authors are grateful to David Stuart for helpful remarks.

\section{References}

[BKKS07] Vladimir Buslaev, Alexander Komech, Elena Kopylova, and David Stuart, On asymptotic stability of solitary waves in a nonlinear Schrödinger equation, arXiv math-ph/0702013, MPI Preprint Nr. 37/2007, http://www.mis.mpg.de/preprints/2005/prepr2007_37.html.

[KK06] Alexander I. Komech and Andrew A. Komech, On global attraction to solitary waves for the KleinGordon equation coupled to nonlinear oscillator, C. R., Math., Acad. Sci. Paris 343 (2006), 111114.

[KK07] Global attractor for a nonlinear oscillator coupled to the Klein-Gordon field, Arch. Ration. Mech. Anal. (2007), accepted. MPI Preprint Nr. 121/2005, http://www.mis.mpg.de/preprints/2005/prepr2005_121.html. 